\title{
Preliminary Sketch of Possible Fixed Point Transformations for Use in Adaptive Control
}

\author{
József K. Tar, János F. Bitó \\ Budapest Tech \\ John von Neumann \\ Faculty of Informatics \\ Inst. of Intelligent Engineering Systems \\ Bécsi út 96/B, Budapest, \\ H-1034, Hungary \\ tar.jozsef@nik.bmf.hu, bito@bmf.hu
}

\author{
László Nádai \\ Budapest Tech \\ Transportation Informatics \\ and \\ Bécsi út 96/B, Budapest, \\ H-1034, Hungary \\ nadai@bmf .hu
}

Telematics Knowledge Center Rua Dr. Antonio Bernardino de Almeida

\author{
José A. Tenreiro Machado \\ Institute of Engineering of Porto \\ Department of
4200-072 Porto,
Portugal \\ jtm@isep.ipp.pt
}

\begin{abstract}
In this paper a further step towards a novel approach to adaptive nonlinear control developed at Budapest Tech in the past few years is reported. Its main advantage in comparison with the complicated Lyapunov function based techniques is that it is based on simple geometric considerations on the basis of which the control task can be formulated as a Fixed Point Problem for the solution of which a Contractive Mapping is created that generates an Iterative Cauchy Sequence for Single Input - Single Output (SISO) systems. Consequently it converges to the fixed point that is the solution of the control task. In the formerly developed approaches for monotone increasing or monotone decreasing systems the proper fixed points had only a finite basin of attraction outside of which the iteration might become divergent. The here sketched potential solutions apply a special function built up of the "response function" of the excited system under control and of a few parameters. This function has almost constant value apart from a finite region in which it has a "wrinkle" in the vicinity of the desired solution that is the "proper" fixed point of this function. By the use of an affine approximation of the response function around the solution it is shown that at one of its sides this fixed point is repulsive, while at the opposite side it is attractive. It is shown, too, that at the repulsive side another, so called "false" fixed point is present that is globally attractive, with the exception of the basin of attraction of the "proper" one. This structure is advantageous because a) no divergence can occur in the iteration, b) the convergence to the "false" value can easily be detected, and c) by using some ancillary tricks in the most of the cases the solution can be kicked from the wrong fixed point into the basin of attraction of the "proper one". In the paper preliminary calculations are presented.
\end{abstract}

\section{Introduction: The Advantages of the GeOMEtRiC WAY OF THINKING}

Until the $1^{\text {st }}$ half of the $20^{\text {th }}$ century the development of Mathematics aimed at serving the needs of natural and technical sciences. In the history of the "quantitative sciences" geometric way of thinking always played pioneering role.

The first attempt to develop mathematics in "axiomatic manner" was done by Euclid of Alexandria, a Greek mathematician in Egypt during the reign of Ptolemy I (323-283 BC) [1]. His method remained the backbone of mathematics even in our days.
The first mathematical means definitely describing quantities with direction, i.e. the quaternions, were introduced by Sir William Rowan Hamilton (1805-1865) [2]. In the $19^{\text {th }}$ century quaternions were generally used for such purposes. For instance, in the first edition of Maxwell's famous Treatise on Electricity and Magnetism quaternions were used for describing the "directed" magnetic and electric fields [3].

The first known appearance of "linear algebra" and the notion of "vector space" is related to Hermann Günther Grassmann (1809-1877), who started to work on the concept from 1832. He published his "Ausdehnungslehre" [4], [5] that normally is referred to as the "theory of extension" or "theory of extensive magnitudes". This work was mainly inspired by Lagrange's "Mécanique analytique" [6]. Grassmann showed that in his algebraic formalization of Geometry the number of possible dimensions can be an arbitrary positive integer.

David Hilbert (1862-1943) extended the concept of the Euclidean Geometry to linear, normed, complete metric spaces in which the norm originates from a scalar product [7]. His invention, the so called Hilbert Space had extreme advantages in Physics and technical sciences since it made it possible to apply a way of geometric thinking with which we became familiar from our childhood in the daily experienced Euclidean Geometry of the reality around us, either using even complex, or only real Hilbert Spaces.

Stefan Banach (1892-1945) introduced the more general concept, the concept of "Banach Spaces", that are linear, normed, complete metric spaces in which the norm not necessarily originates from a scalar product [8]. The great practical advantage of Banach's invention is that by adding various norms to the same mathematical set various complete, linear, normed metric spaces can be obtained that offer a wide basis for elaborating diverse practical variants and solutions pertaining to the essentially same basic idea. Due the completeness of Banach Spaces, each Cauchy Sequence in such a space converges to an element of the space. This property of the Banach Spaces is essentially used in the proof of convergence of the proposed method as it is detailed in the sequel. 
The paper is structured as follows: in Section II the immediate antecedents are briefly surveyed. In Section III some potential new constructions are considered and analyzed. Section IV contains the conclusions of the paper.

\section{IMMEDiATE ANTECEDENTS}

The novel geometric approach is base on the "Excitation Response Scheme". The great majority of the control tasks can be formulated by the use of the concepts of the appropriate "excitation" $Q$ of the controlled system to which it is expected to respond by some prescribed or "desired response" $r^{d}$. The necessary excitation can be computed by the use of some inverse dynamic model $Q=\varphi\left(r^{d}\right)$. Since normally this inverse model is neither complete nor exact, the actual response determined by the system's dynamics, $\psi$, results in a realized response $r^{r}$ that differs from the desired one: $r^{r} \equiv$ $\psi\left(\varphi\left(r^{d}\right)\right) \equiv f\left(r^{d}\right) \neq r^{d}$. It is worth noting that the functions $\varphi()$ and $\psi()$ may contain various hidden parameters that partly correspond to the dynamic model of the system, and partly pertain to unknown external dynamic forces acting on it. Due to phenomenological reasons the controller can manipulate or "deform" the input value from $r^{d}$ so that $r^{r} \equiv \psi\left(r_{*}^{d}\right)$. Other possibility is the manipulation of the output of the rough model as $r^{r} \equiv \psi\left(\varphi^{*}\left(r^{d}\right)\right)$. Normally, for SISO systems the appropriate deformation can be defined as some Parametric Fixed Point Transformations.

The first efforts in the direction of applying uniform structures and procedures in quite different way as it is done in the classic Soft Computing applications were summarized in [9] in which the sizes of the necessary uniform structures used for developing partial, temporal, and situation-dependent models that needed continuous maintaining were definitely determined by the degree of freedom of the system to be controlled. These considerations were based on the modification of the Renormalization Transformation, and were valid only for "increasing systems" in which the "increase" in the necessary response could be achieved by also increasing the necessary excitation, and vice versa. In [10] this idea was systematically extended for Single Input - Single Output (SISO) "increasing" and "decreasing" systems by developing various Parametric Fixed Point Transformations more or less akin to the Renormalization Transformation. By the use of the method of real Singular Value Decomposition (SVD) e.g. [12] this approach has been extended to Multiple Input - Multiple Output (MIMO) sytems [13].

Each of these approaches had the common deficiency that they were apt to become divergent when the basin of attraction was occasionally left by the state variables of the controlled system. This observation induced the need of designing novel fixed point transformations that cannot be divergent, allow easy monitoring of the formation of the actual sequence, and also allows the correction of the convergence problems in a simple way.

The original idea for increasing systems suggested the iteration $x_{n+1}=\frac{x^{d}}{f\left(x_{n}\right)} x_{n} \equiv \theta\left(x_{n} \mid x^{d}\right)$. Really, if $f\left(x_{\star}\right)=x^{d}$ then $\theta\left(x_{\star} \mid x^{d}\right)=x_{\star}$, and it can be expected that from an initial value $x_{0}$ the iteration converges to the fixed point $x_{\star}$.

Fixed point problems in general have the advantageous feature that they can be solved via simple iteration provided that this iteration is convergent. Really, consider the sequence of points $\left\{x_{0}, x_{1}=\Psi\left(x_{0}\right), \ldots, x_{n+1}=\Psi\left(x_{n}\right), \ldots\right\}$ obtained via iteration! Let us suppose that this series converges to some $x_{n} \rightarrow x_{*}$. In order to apply iterations let us consider the set of the real numbers $\Re$ as a linear normed space with the common addition and multiplication with real numbers, and with the absolute value $|\bullet|$ as a norm! It is well known that this space is complete, i.e. it is a Banach Space in which the Cauchy Sequences are convergent. Due to that, using the norm inequality it is obtained that

$$
\begin{gathered}
\left|\Psi\left(x_{*}\right)-x_{*}\right| \leq\left|\Psi\left(x_{*}\right)-x_{n}\right|+\left|x_{n}-x_{*}\right|= \\
=\left|\Psi\left(x_{*}\right)-\Psi\left(x_{n-1}\right)\right|+\left|x_{n}-x_{*}\right| .
\end{gathered}
$$

It is evident from (1) that if $\Psi$ is continuous then $\Psi\left(x_{*}\right)=$ $x_{*}$, i.e. $x_{*}=x_{\star}$, that is the desired fixed point is found by the iteration because in the right hand side of (1) both terms converge to 0 as $x_{n} \rightarrow x_{*}$. (It is worth noting that besides divergence, false convergence may also occur.)

The next question is giving the necessary or at least a satisfactory condition of this convergence. It also is evident that for this purpose contractivity of $\Psi(\bullet)$, i.e. the property that $|\Psi(a)-\Psi(b)| \leq K|a-b|$ with $0 \leq K<1$ is satisfactory since it leads to a Cauchy series $\left(\left|x_{n+L}-x_{n}\right| \rightarrow 0 \forall L \in \mathrm{N}\right)$ :

$$
\begin{gathered}
\left|x_{n+L}-x_{n}\right|=\left|\Psi\left(x_{n+L-1}\right)-\Psi\left(x_{n-1}\right)\right| \leq \ldots \\
\leq K^{n}\left|x_{L}-x_{0}\right| \rightarrow 0 \text { as } n \rightarrow \infty
\end{gathered}
$$

For guaranteeing the contractivity of a differentiable $\Re \rightarrow \Re$ function $\Psi(\bullet)$ proper limitation on the absolute value of the derivative $\left|\Psi^{\prime}\right| \leq K<1$ is satisfactory since

$$
\begin{gathered}
|\Psi(a)-\Psi(b)|=\left|\int_{a}^{b} \Psi^{\prime}(x) d x\right| \leq \\
\quad \leq \int_{a}^{b}\left|\Psi^{\prime}(x)\right| d x \leq K|a-b|
\end{gathered}
$$

that means that if $\Psi$ is flat enough around the fixed point the iteration will converge to it. Since

$$
\frac{d \theta}{d x}=-\frac{x^{d} x f^{\prime}(x)}{f(x)^{2}}+\frac{x^{d}}{f(x)}
$$

the situation of $-1<\theta^{\prime}\left(x_{\star}\right)=1-\frac{x}{x^{d}} f^{\prime}\left(x_{\star}\right)<1$ can be achieved if $x \approx x^{d}$, and $0<f^{\prime}\left(x_{\star}\right)$ is small enough. This latter condition can be satisfied by choosing very flat initial model $\varphi$ if $\psi(\bullet)$ is not singular. For $f^{\prime}<0$ this iteration scarcely can converge. For negative system in [10] the following iteration was proposed with small $\zeta>0$ :

$$
x_{n+1}=\frac{f\left(x_{n}\right)+\zeta\left(f\left(x_{n}\right)-x^{d}\right)}{f\left(x_{n}\right)} x_{n} \equiv \vartheta\left(x_{n} \mid x^{d}\right) .
$$

Evidently $\vartheta\left(x_{\star} \mid x^{d}\right)=x_{\star}, \vartheta^{\prime}=1+\zeta-\zeta \frac{x^{d}}{f(x)}+\zeta \frac{x^{d} x}{f(x)^{2}} f^{\prime}$, so $\vartheta^{\prime}\left(x_{\star}\right)=1+\zeta \frac{x}{x^{d}} f^{\prime}\left(x_{\star}\right)$. If $0<\zeta<1, x \approx x^{d}, f^{\prime}\left(x_{\star}\right)<0$, and $\left|f\left(x_{\star}\right)^{\prime}\right|$ is small enough this iteration will converge to 
the fixed point $x_{\star}$, too. To evade numerical problems with $x^{d}=0$ and $f\left(x_{\star}\right)=0$ an additional "shift parameter" $D$ can be introduced into (5) with the properties as follows:

$$
\begin{gathered}
\vartheta(x \mid \zeta, D):=\frac{f(x)+D+\zeta\left(f(x)-x^{d}\right)}{f(x)+D} x, \\
\vartheta\left(x_{\star} \mid \zeta, D\right)=x_{\star}, \\
\frac{d \vartheta(x \mid \zeta, D)}{d x}=1+\zeta \frac{f(x)-x^{d}}{f(x)+D}+ \\
+x \zeta f^{\prime}(x) \frac{f(x)+D-\left(f(x)-x^{d}\right)}{\left.(f(x)+D)^{2}\right)^{\prime}} \\
{\left[\frac{d \vartheta(x \mid \zeta, D)}{d x}\right]_{x=x_{\star}}=1+\frac{\zeta f^{\prime}\left(x_{\star}\right) x_{\star}}{x^{d}+D}}
\end{gathered}
$$

The convergence of (6) may happen. However, certain illustrative examples of the possibility of convergence to "false values" and the relatively complicated derivatives in (6) that makes not very easy to find proper $D$ and $\zeta$ parameters made it actual to find other transformations that have more lucid nature.

In the case of SISO systems this can be done by introducing two parameters ad $D_{-}$, and $\Delta_{-}$in the case of monotone increasing functions indicated by a very simple geometric picture based on geometrically similar triangles. For monotone increasing or decreasing SISO systems, by introducing function $g$ as defined below three further combinations were invented to which figures quite similar to the original one belong in [11], and were used in control of a SISO paradigm [15]. (In some of these functions instead of the parameter $\Delta_{-}$ the parameter $\Delta_{+}$is present.)

$$
\begin{gathered}
h\left(x \mid x^{d}, D_{-}, \Delta_{-}\right):= \\
=\frac{\left(x^{d}-\Delta_{-}\right)\left(x-D_{-}\right)}{f(x)-\Delta_{-}}+D_{-}, \\
h\left(x_{\star} \mid x^{d}, D_{-}, \Delta_{-}\right)=x_{\star}, \\
h^{\prime}=\frac{\left(x^{d}-\Delta_{-}\right)\left(f(x)-\Delta_{-}-f^{\prime}(x)\left(x-D_{-}\right)\right)}{\left(f(x)-\Delta_{-}\right)^{2}}, \\
h^{\prime}\left(x_{\star} \mid x^{d}, D_{-}, \Delta_{-}\right)=1-f^{\prime}\left(x_{\star}\right) \frac{x_{\star}-D_{-}}{x^{d}-\Delta_{-}}
\end{gathered}
$$

It is worth noting that if the rough dynamic model consists of an affine function the desired response as $\varphi\left(r^{d}\right)=a r^{d}+b$, in SISO systems, besides the parameters $a$ and $b$ the control is defined by further simple parameters as $D_{-}, \Delta_{-}$, or $\Delta_{+}$, it is very easy to find the proper settings by computer simulations. To show that it is enough to observe the derivatives in the fixed point $x_{\star}$. For instance, consider e.g. $h^{\prime}\left(x_{\star} \mid x^{d}, D_{-}, \Delta_{+}\right)=$ $1-f^{\prime}\left(x_{\star}\right) \frac{x_{\star}-D_{-}}{x^{d}-\Delta_{+}}$! If $\left|x_{\star}\right| \ll\left|D_{-}\right|,\left|x^{d}\right| \ll\left|\Delta_{+}\right|$, and $D_{-}$and $\Delta_{+}$are of the same order of magnitude then $\frac{x_{\star}-D_{-}}{x^{d}-\Delta_{+}} \approx \frac{D_{-}}{\Delta_{+}}=$ const., almost independently of $x^{d}$ and $x_{\star}$. Furthermore, if $|a|$ is small enough the $\left|h^{\prime}\right|<1$ condition of convergence almost surely can be met. So the control parameters can "experimentally" be set via simulations by choosing small $a$, and comparably big $D_{-}, \Delta_{+}$. Later the absolute values of these latter two parameters can be decreased to achieve more and more sensitive control until reaching the limits of stability. If necessary, $|a|$ can also be decreased. (Evidently similar considerations can be done for the parameters of the other cases discussed above.)

The above fixed point transformations have the deficiency that even for "not very extremely behaving" $f(x)$ functions they may lead to divergence since the behavior of their derivatives apart from the close vicinity of $x_{\star}$ cannot well be seen and controlled. To evade this problem in the present paper the posible introduction of novel fixed point transformations is considered.

\section{PRoposal FOR FURTher FiXed-Point TRANSFORMATIONS}

Let $A \geq 0, n>0$ even natural number, and $K$ be an arbitrary value. Consider the function defined by (8). As it is well illustrated by Fig. 1, if an increasing function can well be approximated around the fixed point $x_{\star}$ by an affine mapping, then in the $\left(x_{\star},-K\right]$ region $\left|G^{\prime}\right|<1$ and the $x=-K$ value serves as an attractive fixed point of the $x_{n+1}=G\left(x_{n} \mid x^{d}\right)$ iteration. It also is evident that $x=-K$ is also attractive for the $x>-K$ starting points. It is important that the $x_{\star}$ point serves as an attractor for the starting $x$ values that are sligtly smaller than $x_{\star}$.

$$
\begin{aligned}
& G\left(x \mid x^{d}\right):=(x+K) \exp \left(-A\left[f(x)-x^{d}\right]^{n}\right)-K, \\
& F \text { orf }\left(x_{\star}\right)=x^{d}: G\left(x_{\star} \mid x^{d}\right)=x_{\star}, \\
& G\left(-K, x^{d}\right)=-K, \\
& G^{\prime}\left(x \mid x^{d}\right)=\frac{\left\{1-\frac{A n(x+K)}{\left[f(x)-x^{d}\right]^{1-n}} f^{\prime}(x)\right\}}{\exp \left(A\left[f(x)-x^{d}\right]^{n}\right)}, \\
& G^{\prime}\left(x_{\star} \mid x^{d}\right)=1, G^{\prime}\left(-K \mid x^{d}\right)= \\
& =\exp \left(-A\left[f(-K)-x^{d}\right]^{n}\right) \leq 1
\end{aligned}
$$

However, if an $x_{0}$ initial value is considerably smaller than $x_{\star}$ the first iterative step will map it to a point $x_{1}>-K$, so these points also belong to the basin of attraction of $x=-K$. It is evident that if $f(x)$ is not too "extreme", the proposed iteration cannot be divergent: it must converge either to $x_{\star}$ or to $-K$.

The prosed iteration has the great advantage as follows: a) the $x=-K$ point can be referred to as an "improper fixed point" that is well known, and independent of the actual function $f(x)$, of the other parameters as $A, n, x^{d}$, and the initial point of the iteration $x_{0}$; b) the "proper fixed point" always exists, it is the solution that is sought for, it is independent of the parameters $K, n$, it exceptionally depends on $f(x)$ and $x^{d}$, and surely has a bounded basin of attraction at one of its sides (in Fig. 1 at its left hand side); c) the location of the two existing fixed points is independent of the parameters $A$ and $n$, and since the graph of the function $y=x$ is the tangent of that of $G\left(x \mid x^{d}\right)$ at $x_{\star}$, the parameters $A$ and $n$ mainly influence the width of the basin of attraction of $x_{\star}$ at its appropriate side, as well as the speed of convergence. This fact is well illustrated by Fig. 2 describing the convergence of the iteration starting from the $x_{0}=0$ initial value. It can well be observed, too, that the convergence slows down in the vicinity of $x_{\star}$ where $\left|G^{\prime}\right|$ approaches 1 . If $x_{0}$ is too far from $x_{\star}$ instead of any divergence a convergence can be observed to $-K$ (Fig. 3).

Evidently, like in the case of the previous fixed-point transformations typical cases can be proposed for this function $G\left(x \mid x^{d}\right)$ as follows: a) for $f^{\prime}>0$ (increasing functions): since $A>0$, if $x+K<0, f(x)<x^{d}$, then $\left|G^{\prime}\right|<1$ can be 


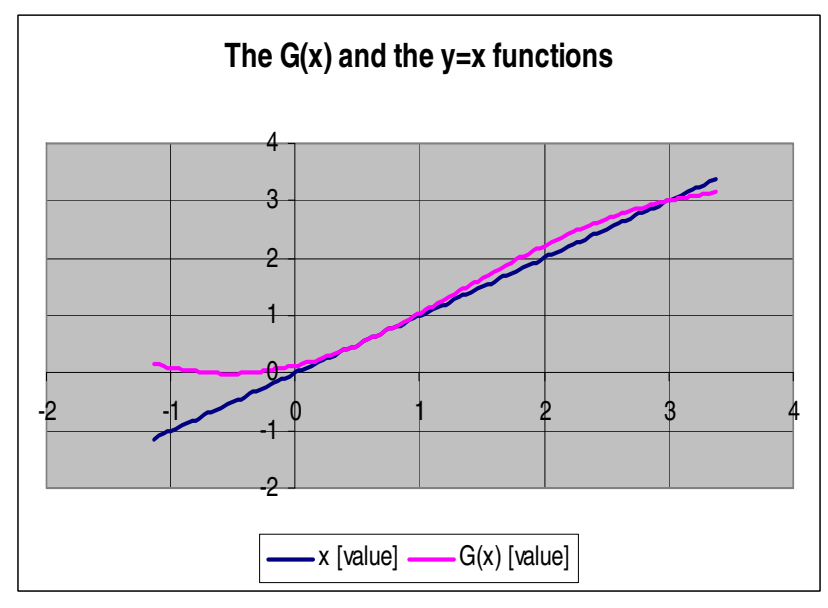

Fig. 1. The curve of the graph of function $G\left(x, x^{d}\right)$ defined in (8) for $n=2$, $A=5 \times 10^{-3}, K=-3$ in the case of an affine function $f(x)=b x+c$ with $b=5, c=3$, and $x^{d}=6$
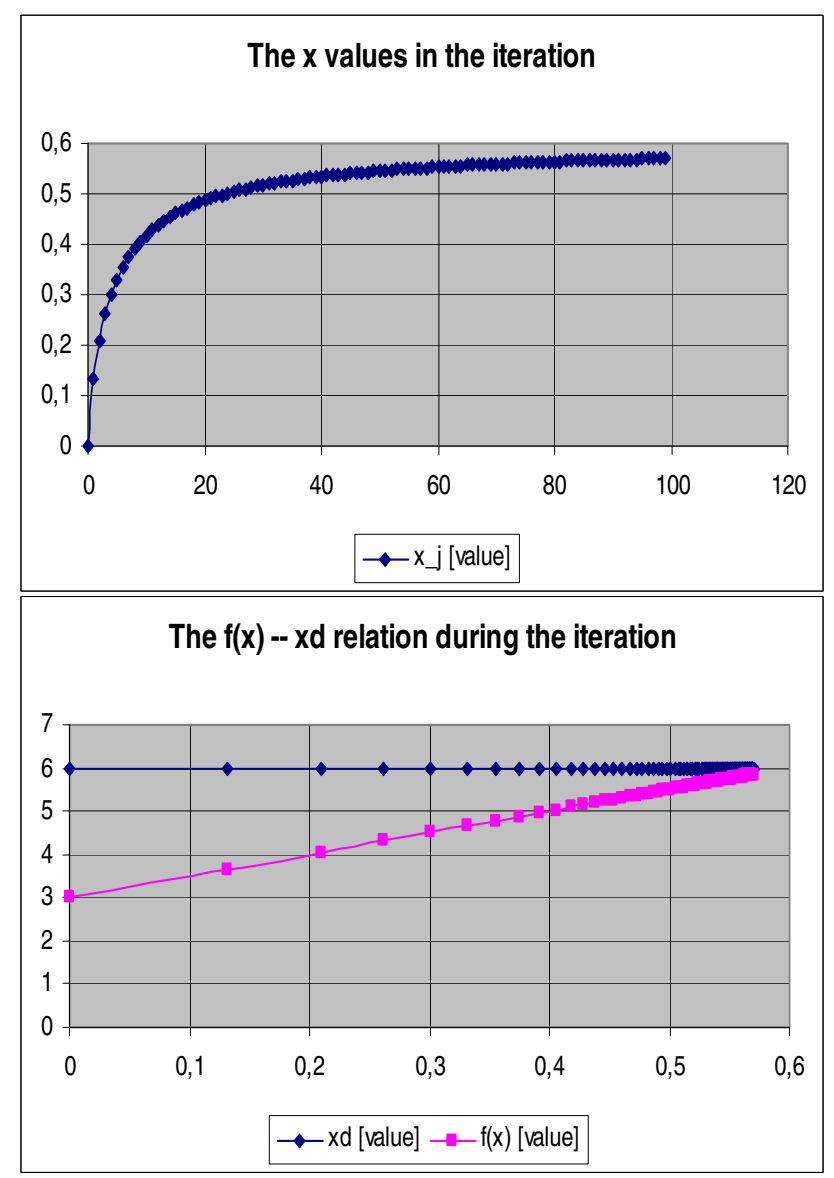

Fig. 2. The "proper" convergence from the initial value $x_{0}=0$ in the case of the function depicted in Fig. 1
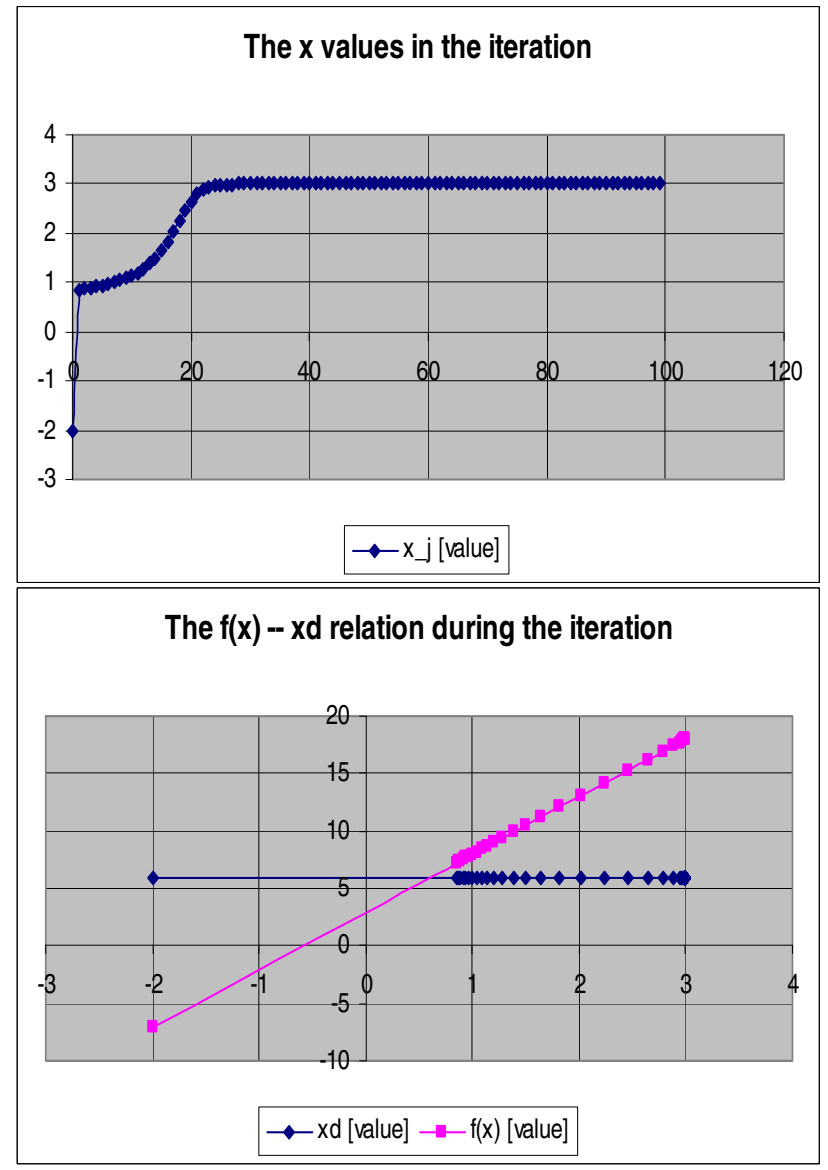

Fig. 3. The "false" convergence from the initial value $x_{0}=-2$ in the case of the function depicted in Fig. 1

achieved in the vicinity of $x_{\star}$; b) for $f^{\prime}>0$ (increasing functions): since $A>0$, if $x+K>0$, and $f(x)>x^{d}$, then $\left|G^{\prime}\right|<1$ can be achieved in the vicinity of $x_{\star}$; c) for $f^{\prime}<0$ (decreasing functions): since $A>0$, if $x+K<0$, and $f(x)>x^{d}$, then $\left|G^{\prime}\right|<1$ can be achieved in the vicinity of $x_{\star} ;$ c) for $f^{\prime}<0$ (decreasing functions): since $A>0$, if $x+K>0, f(x)<x^{d}$, then $\left|G^{\prime}\right|<1$ can be achieved in the vicinity of $x_{\star}$.

It is worth noting that the property $G^{\prime}\left(x_{\star} \mid x^{d}\right)=1$ may be inconvenient for control purposes because it may slow down the iteration's convergence to $x_{\star}$. (If the graph of $G$ is not flat enough very much steps with almost ignorable improvement may be obtained.) This problem cannot be cured plausible tricks as e.g. by dividing the coefficient of the term $(x+K)$ into two parts as e.g. for $B \in(-1,1)$ in

$$
H\left(x \mid x^{d}\right):=(x+K)\left[(1-B)+B e^{-A\left[f(x)-x^{d}\right]^{n}}\right]-K .
$$

The derivative at $x_{\star}$ will be 1 again since $n$ is positive even number 


$$
\begin{aligned}
& H^{\prime}\left(x \mid x^{d}\right)=(1-B)+B e^{-A\left[f(x)-x^{d}\right]^{n}}+ \\
& +(x+K) \times \\
& \times\left[-A B e^{-A\left[f(x)-x^{d}\right]^{n}} n\left[f(x)-x^{d}\right]^{n-1} f^{\prime}(x)\right] .
\end{aligned}
$$

Of course, for achieving similar qualitative behavior various other mappings can also be chosen with a common structure more or less similar to that of (8). For instance, consider the following simple possibilities as (11), or for positive even integer $n$ the expression defined in (12)! For their use one can again distinguish between "increasing" and "decreasing" functions with the initial points $f\left(x_{0}\right)>x^{d}$ or $f\left(x_{0}\right)<$ $x^{d}$. Due to room limitations no more figures will be made to illustrate their operation individually. Instead of that a possible utilization of their common feature, that is the simple possibility for the detection of the convergence to the "false" fixed-point will be illustrated in the case of (11).

$$
\begin{gathered}
\hat{G}\left(x \mid x^{d}\right):=(x+K) \exp \left(-A\left|f(x)-x^{d}\right|\right)-K, \\
F \operatorname{ror} f\left(x_{\star}\right)=x^{d}: \hat{G}\left(x_{\star} \mid x^{d}\right)=x_{\star}, \\
\hat{G}\left(-K, x^{d}\right)=-K, \\
\hat{G}^{\prime}\left(x \mid x^{d}\right)=\frac{1-A(x+K) \operatorname{sign}\left[f(x)-x^{d}\right] f^{\prime}(x)}{\exp \left(A\left|f(x)-x^{d}\right|\right)}, \\
\hat{G}^{\prime}\left(x_{\star \pm} \mid x^{d}\right)=1 \mp A\left(x_{\star}+K\right) f^{\prime}\left(x_{\star}\right) \\
\hat{G}^{\prime}\left(-K \mid x^{d}\right)=\exp \left(-A\left|f(-K)-x^{d}\right|\right), \\
\tilde{G}\left(x \mid x^{d}\right):=\frac{(x+K)}{1+\left[A\left(f(x)-x^{d}\right)\right]^{n}}-K, \\
F \operatorname{For} f\left(x_{\star}\right)=x^{d}: \tilde{G}\left(x_{\star} \mid x^{d}\right)=x_{\star}, \\
\tilde{G}\left(-K, x^{d}\right)=-K, \\
\tilde{G}\left(x \mid x^{d}\right)=\frac{1}{1+\left[A\left(f(x)-x^{d}\right)\right]^{n}} \times \\
\times\left[1-\frac{A n(x+K)\left[A\left(f(x)-x^{d}\right)\right]^{n-1} f^{\prime}(x)}{1+\left[A\left(f(x)-x^{d}\right)\right]^{n}}\right] \\
\tilde{G}\left(x_{\star} \mid x^{d}\right)=1, \\
\tilde{G}\left(-K \mid x^{d}\right)=\frac{1}{1+\left[A\left(f(-K)-x^{d}\right)\right]^{n}} \leq 1 .
\end{gathered}
$$

Instead of the simple sequence $x_{n+1}=\hat{G}\left(x_{n} \mid x^{d}\right)$ a little bit modified one casually referred to as "smart iterative sequence" is illustrated. According to the syntax of Visual Basic it has the following simple code:

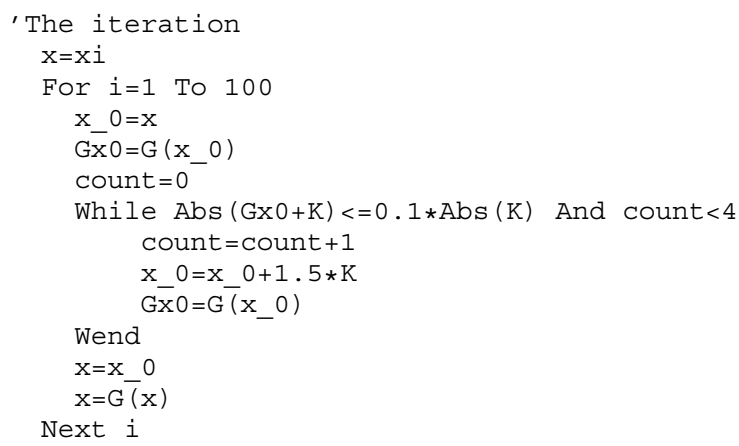

In the above structure the true logical value of fulfilment of the condition $\left|G\left(x_{0}\right)-(-K)\right| \leq 0.1|K|$ is interpreted in the way as follows: "The iteration is in the vicinity $\backslash$ probably converges to the false fixed point $-K$ ". In this case an attempt is done for pushing the initial point "behind the solution" $x_{\star}$. If no such
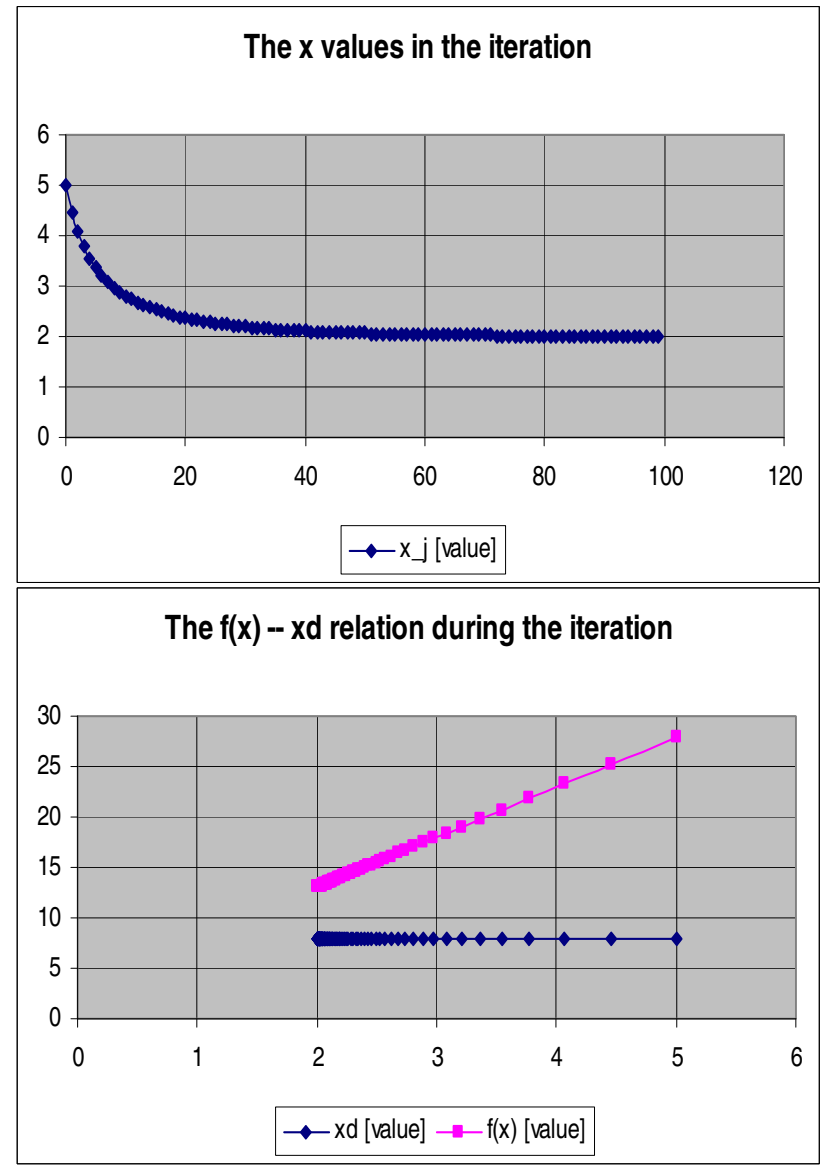

Fig. 4. The "false" convergence of the "common" iteration starting from the initial value $x_{0}=5$ in the case of the function $\hat{G}$ with parameters $A=10^{-1}$, $K=-2$, and $x^{d}=8$ for the function $f(x)=b x+c$ with $b=5, c=3$

problem is detected the common iteration is going on. As it is illustrated by Fig. 4 the "common iteration" approaches the "false" fixed point.

However, according to Fig. 5 the "smart" version of the iteration pushes back the first point that too closely approaches $-K$, and following that it converges to the good solution.

\section{Conclusions}

In this paper novel fixed point transformations were investigated and proposed for purposes of adative control on the basis of some "geometric way of thinking". They have the advantage that under normal conditions they introduce a single wrinkle into an almost constant function. Within the wrinkle an almost globally attractive fixed point of the iteration defined by this function is located, and an another fixed point that has a narrow basin of attraction at its one side, and repulsive in its opposite side is located. It was shown by using an affin approximation of the response function of a MIMO system that the attributes of the convergence to the "false" fixed point can easily be recognized and evaded by a simple modification of the common iterative process. It is expected that this simple method can well be supported by a simple rule basis for practical use. 


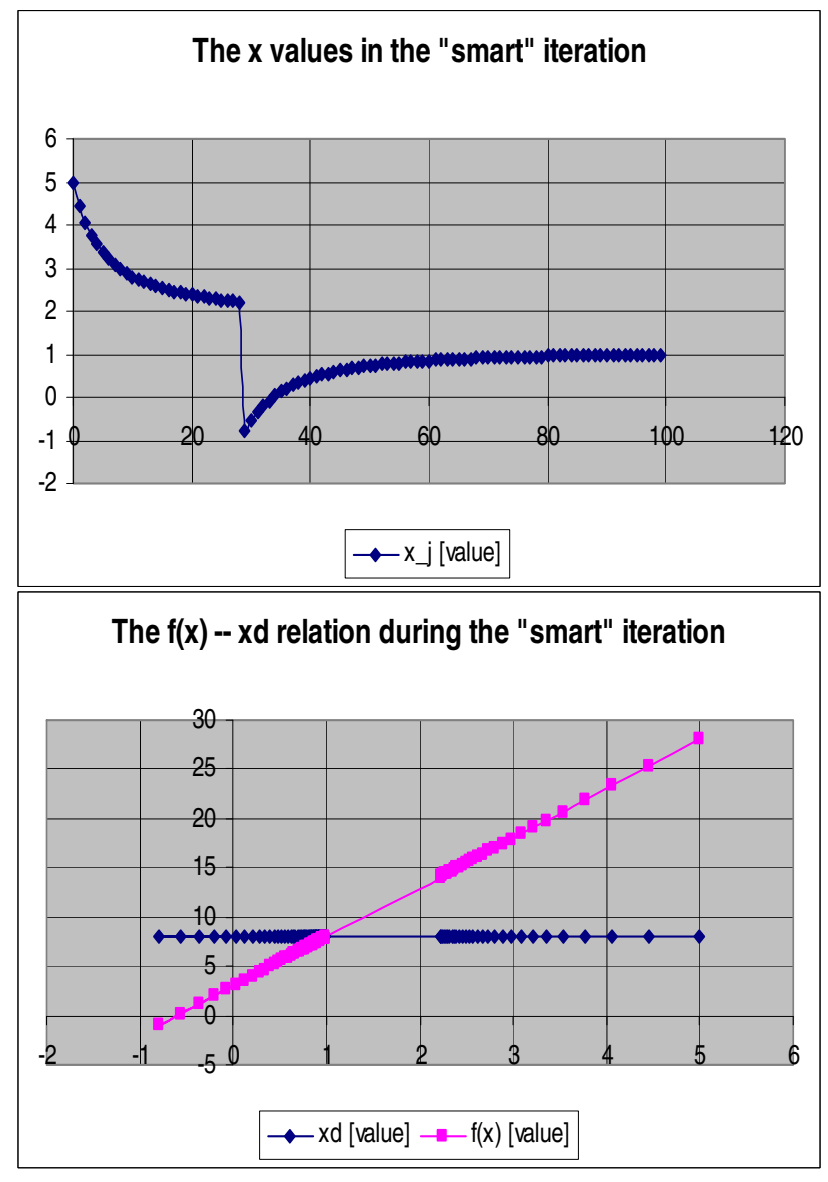

Fig. 5. The good convergence of the "smart" iteration starting from the initial value $x_{0}=5$ in the case of the function $\hat{G}$ with parameters $A=10^{-1}$, $K=-2$, and $x^{d}=8$ for the function $f(x)=b x+c$ with $b=5$, and $c=3$

In the future we plan to investigate nonlinear control tasks in which the variable here denoted by $x$ corresponds to some integer or fractional order derivative of the state variable of some physical system that can directly be manipulated by appropriate physical agents as e.g. the acceleration can be manipulate by forces. If the nominal motion of the system to be controlled is considerably slower than the necessary variation of $x_{n}$ in the iterations then the idea of Complete Stability [14] can be applied in the control in quite similar manner as dynamic Cellular Neural Networks can be able to analyze pictures slowly varying in time.

\section{ACKNOWLEDGMENT}

The authors gratefully acknowledge the support by the Hungarian National Research Fund (OTKA) within the project No. K063405. This research was also supported by the National Office for Research and Technology (NKTH) in Hungary using the resources of the Research and Technology Innovation Fund within the project No. RET-10/2006 and that of the bilateral Hungarian-Portuguese S\&T Program No. PT-12/07.

\section{REFERENCES}

[1] T.L. Heath: "The Thirteen Books of Euclid's Elements", Vol. 1, 2nd ed. (1956) New York: Dover Publications

[2] W.R. Hamilton: "On a General Method in Dynamics", Philosophical Transaction of the Royal Society Part I (1834) p.247-308; Part II (1835) p 95-144. (From the collection Sir William Rowan Hamilton (1805-1865): Mathematical Papers edited by David R. Wilkins, School of Mathematics, Trinity College, Dublin 2, Ireland. (2000); also reviewed as On a General Method in Dynamics)

[3] J. Clerk Maxwell: "A Treatise on Electricity and Magnetism", $3^{\text {rd }}$ ed., vol. 2. Oxford: Clarendon, 1892, pp.68-73.

[4] H. Grassmann: "Die lineare Ausdehnungslehre", Leipzig: Wiegand, 1844 (English translation, by Lloyd Kannenberg: "A new branch of mathematics", Chicago: Open Court, 1995)

[5] H. Grassmann: "Die Ausdehnungslehre, vollständig und in strenger Form bearbeitet", Berlin: Enslin, 1862 (English translation, by Lloyd Kannenberg: "Extension Theory", American Mathematical Society, 2000)

[6] Joseph-Louis Lagrange: "Mécanique Analytique (Analytical Mechanics)", (4. ed., 2 vols. Paris: Gauthier-Villars et fils, 1888-1889. (First Edition in 1788)

[7] M. Hallett and U. Majer: "David Hilbert's Lectures on the Foundations of Geometry: 1891-1902", Springer-Verlag Berlin Heidelberg, 2004

[8] R. Kałuża, W. Woyczynski, A. Kostant: "Through a Reporter's Eyes: The Life of Stefan Banach", Birkhauser, 1996.

[9] J.K. Tar, I.J. Rudas, Á. Szeghegyi and K. Kozłowski: Novel Adaptive Control of Partially Modeled Dynamic Systems, Lecture Notes in Control and Information Sciences, Springer Berlin/Heidelberg, Robot Motion and Control: Recent Development, Part II - Control and Mechanical Systems, Ed. Krzysztof Kozlowski, Volume 335 / 2006, pp. 99 111, ISBN: 1-84628404-X.

[10] J.K. Tar: "Extension of the Modified Renormalization Transformation for the Adaptive Control of Negative Definite SISO Systems. Proc. of the 2nd Romanian-Hungarian Joint Symposium on Applied Computational Intelligence (SACI 2005), May 12-14, 2005, Timişoara, Romania, pp. 447457.

[11] J.K. Tar, I.J. Rudas: "Geometric Approach to Nonlinear Adaptive Control" - Tutorial, in the Proc. of the 4th International Symposium on Applied Computational Intelligence and Informatics (SACI 2007), May 17-18, 2007, Timişoara, Romania, pp. 9-23

[12] G.W. Stewart: "On the early history of singular value decomposition", Technical Report TR-92-31, Institute for Advanced Computer Studies, University of Mariland, March 1992.

[13] J.K. Tar: "Fixed Point Transformations as Simple Geometric Alternatives in Adaptive Control", Proc. of the $5^{t h}$ IEEE International Conference on Computational Cybernetics (ICCC 2007), October 19-21, 2007, Gammarth, Tunis, pp. 19-34

[14] T. Roska: Development of Kilo Real-time Frame Rate TeraOPS Computational Capacity Topographic Microprocessors. Plenary Lecture at 10th International Conference on Advanced Robotics (ICAR 2001), Budapest, Hungary, August 22-25, 2001.

[15] J.K. Tar, I.J. Rudas, \& B. Pátkai: "Comparison of Fractional Robustand Fixed Point Transformations-Based Adaptive Compensation of Dynamic Friction", under appearance in Journal of Advanced Computational Intelligence and Intelligent Informatics, Vol.11 No.9, 2007 